\title{
A Simple, Fast, and Safe Mediator for Congestion Management
}

\author{
Kei Ikegami, ${ }^{1}$ Kyohei Okumura, ${ }^{1}$ Takumi Yoshikawa ${ }^{1}$ \\ ${ }^{1}$ Graduate School of Economics, The University of Tokyo \\ \{ikegami-kei0120, k-okumura, takumi-yoshikawa157\}@g.ecc.u-tokyo.ac.jp
}

\begin{abstract}
Congestion is a severe problem in cities. A large population with little information about each other's preferences hardly reaches equilibrium and causes unexpected congestion. Controlling such congestion requires us to collect information dispersed in the market and to coordinate actions among agents. We aim to design a mediator that a) induces a game with high social welfare in equilibrium, b) computes an equilibrium efficiently, c) works without common prior, and d) performs well even when only some of the agents in the market use the mediator. We propose a mediator based on a version of best response dynamics (BRD). We prove that, in a simple setting with two resources, "good behavior" (reporting truthfully and following the recommendation) forms an (approximate) ex-post Nash equilibrium in the mediated game; in the equilibrium, the welfare is close to the first-best when preferences diverge enough. Furthermore, under a certain behavioral assumption, those who are not using the mediator can always enjoy non-negative payoff gain by joining it even without the full participation of others. Additionally, our experimental results suggest that such results remain valid for more general settings.
\end{abstract}

\section{Introduction}

Congestion is a severe problem in cities. For example, traffic congestion not only impedes the smooth transfer of inhabitants (Kreindler 2018) or causes air pollution (Gibson and Carnovale 2015), but it also increases people's mental burden (Anderson et al. 2016). Congestion in public transportation also decreases productivity, as it results in many delays and accidents. The social cost caused by congestion in Tokyo's railway is estimated at $\$ 3.03$ billion per year (Okano, Ota, and Hirota 2017; NAVITIME 2018). Other examples of congestion include those in museums, amusement parks, tourist spots, or restaurants. Without a doubt, a practical solution to such congestion is urgently needed.

The crowded markets are usually large, and then, each agent may know little to nothing about each other's preferences, making it difficult for agents to reach equilibrium, which causes unexpected congestion. Congestion games are extensively studied in algorithmic game theory literature,

Copyright (c) 2020, Association for the Advancement of Artificial Intelligence (www.aaai.org). All rights reserved. but many papers consider a complete information setting, which seems to be unrealistic in our context. Even if we admit the complete information, the coordination problem still exists: there may be multiple equilibria, and agents will have difficulty agreeing on which one to play. It is also possible that agents will have difficulty computing equilibrium, as they are boundedly rational. Additionally, previous work has shown that agents following a simple learning algorithm may not reach equilibrium (Palaiopanos, Panageas, and Piliouras 2017). In any case, we cannot always expect agents in the crowded market to play an equilibrium.

In order to mitigate the congestion, it is crucial to collect information from agents and coordinate their actions. It is well known that Nash equilibria are often near-optimal in congestion games with complete information under relatively weak assumptions; hence, it is worth investigating how we implement such equilibria. To this end, we introduce a mediator to the large crowded market.

The mediator listens to the reports from agents and sends back individual recommendations. For the moment, it may be helpful to think of the mediator as a web application. Each agent tells the mediator his preference over resources and his opportunity cost (i.e., the degree of congestion aversion). Given this reported type profile, the mediator computes a Nash equilibrium in the induced complete information game via best response dynamics (BRD); then, the mediator recommends that each agent take action in the computed equilibrium. We design the mediator so that good behavior (opt-in the mediator, report their type truthfully, and follow the recommendation) forms an approximate ex-post Nash equilibrium. By this property, the mediator can guide people to the state in which each agent can use his preferred resource with reduced congestion.

One notable feature of our mediator is that its power is very weak: it cannot compel obedience from agents, and agents can opt-out of the mediator, misreport their types, and ignore the recommendation if they want. This property makes it easy to introduce the mediator to the market.

Our mediator differs from the existing countermeasures for congestion. First, peak-load pricing (Vickrey 1963) is the most popular and influential way of handling congestion, but in practice, institutional barriers or regulations of- 


\begin{tabular}{c|cccc}
\hline & Simplicity & Running-time & Class of Games & Safe Participation \\
\hline PRESL & Parameter tuning & Exponential in & Aggregative & \\
(Cummings et al. 2015) & Random & \# of resources & & \\
P-BR & Parameter tuning & Polynomial in & Congestion & \\
(Kearns et al. 2015) & Random & \# of agents and resources & Congestion w/ & Yes \\
CRAB & No tuning & Polynomial in & individual preference & \# of agents and resources
\end{tabular}

Table 1: Comparison of the mediators. Note that "Random" means random mechanism based on differential privacy techniques; CRAB is almost deterministic except that ties are broken uniformly at random (see Algorithm 1). "?" appears in the last column because the listed previous work does not consider the existence of outsiders.

ten prevent such pricing. For example, public transportation fares are often regulated by law and hard to change flexibly. Our mediator does not need a monetary transfer so it is more widely applicable. Second, moral suasion is sometimes used for energy conservation in peak-demand hours, but empirical studies show that its effect, particularly the long-run effect, is ambiguous (Allcott and Rogers 2014; Ito, Ida, and Tanaka 2018). By contrast, our mediator has an incentive guarantee. Third, the congestion information is disclosed to control the crowd (e.g., Google Maps). While each agent changes his behavior individually under such information, our mediator alters the behavior of agents as a whole. Moreover, our mediator recommends action to each agent; this solves the coordination problem and helps boundedly rational agents to play equilibrium. In summary, our mediator is supposed to be more widely applicable than peak-load pricing and more effective than moral suasion and naive information disclosure.

\section{Our results}

For theoretical analysis, we first focus on a simple setting with two resources where agents have the same opportunity cost, and both resources have the same throughput rate. We show that good behavior forms an approximate ex-post Nash equilibrium where the approximation rate tends to zero as the market gets large. Secondly, those who opt-out the mediator, or outsiders, can enjoy nonnegative payoff gain by joining the mediator even when only some of the agents use the mediator if all outsiders choose their favorites. We also generalize these results to the case where agents have different opportunity costs, and resources differ in the throughput rates.

Furthermore, we conduct a welfare analysis. We introduce the notion of the price of mediation (POM), which is the ratio between the welfare attained in the equilibrium induced by the mediator and the first-best. We compute the POM explicitly; in the large market, POM attains 1 at best, and it keeps close to 1 as long as preferences diverge enough. (NB: POM is 1 iff the mediated equilibrium attains the first-best.)

We also conduct multiple kinds of experiments. Our first set of experiments shows that the mediator works well in more general settings. Even when there are more than two resources and agents have heterogeneous opportunity costs, the approximation rate of ex-post Nash equilibrium is bounded by the value inferred from the theoretical results on simple cases.
Our experiments also confirm that our mediator does satisfy the safe participation property in the general cases. Moreover, in the experiments, more outsiders can enjoy strictly positive payoff gain by joining the mediator as a) the larger fraction of people use the mediator and $b$ ) the market gets larger. These results suggest that our mediator performs better in larger markets.

Our second set of experiments investigates the effect of different specifications of BRD. In the usual definition of $\mathrm{BRD}$, the initialization and the rule to pick a mover at each step are not defined. There are several possible ways of implementation. We observe that our specification of BRD outperforms other versions both in the realized social welfare and in the computation time.

\section{Our contribution}

This paper is directly motivated by previous work that has proposed weak mediators, P-BR (Kearns et al. 2015) and PRESL (Cummings et al. 2015); both are applicable to congestion games. They rely on algorithms based on differential privacy techniques. Though their mediators have various favorable theoretical properties, we face difficulties when implementing it in practice, as we will discuss shortly. We aim at an implementation-oriented mediator.

Our conceptual contribution is the introduction of a mediator named Credible Recommender based on Agent-made Billboard (hereafter called CRAB), based on a simple BRD. The previous work argues that a mediator who naively computes a Nash equilibrium of the complete information game induced by reported types may fail (Kearns et al. 2014). In order to prevent such failure, it is crucial to ensure that each agent can hardly affect the output of the mediator by misreporting; this is why they use differentially private algorithms. However, we show that, in congestion games (with player-specific utilities), the mediator based on a simple BRD performs well. CRAB has the following distinctive features (see also Table 1):

Simplicity CRAB is simple in the following two senses: First, given a reported type profile, CRAB returns an equilibrium action profile with certainty. In contrast, both P-BR and PRESL return a non-equilibrium action profile, which can result in poor social welfare with a small probability. This property is sometimes problematic when we persuade practitioners to use the mediator. Second, unlike CRAB, we 
need hyperparameter tuning for P-BR and PRESL. This point also makes them hard to deploy.

Fast computation The running time of CRAB is polynomial in both the number of agents and resources (Proposition 1) since it relies on a simple $\epsilon$-BRD. In contrast, PRESL takes time exponential in the number of resources. P-BR's running time is also polynomial, but we show through experiments that ours is more efficient. Table 2 shows the running time of CRAB and P-BR.

\begin{tabular}{c|cc}
\hline$(n, k)$ & CRAB & P-BR \\
\hline$(100,5)$ & $0.003 \mathrm{~s}$ & $2.326 \mathrm{~s}$ \\
$(100,10)$ & $0.005 \mathrm{~s}$ & $3.522 \mathrm{~s}$ \\
$(200,5)$ & $0.013 \mathrm{~s}$ & $29.332 \mathrm{~s}$ \\
$(200,10)$ & $0.022 \mathrm{~s}$ & $71.988 \mathrm{~s}$ \\
$(500,5)$ & $0.0647 \mathrm{~s}$ & $466.132 \mathrm{~s}$ \\
\hline
\end{tabular}

Table 2: CRAB vs. P-BR: Running time comparison. $(n, k)$ is a pair of the number of agents $n$ and the number of resources $k$.

Generality CRAB can deal with singleton congestion games with player-specific utilities (Milchtaich 1996). This game class is a subclass of multi-dimensional aggregative games, but a superclass of simple singleton congestion games (Ieong et al. 2005), and general enough to treat allocation problems with negative externality.

Safe participation When we consider introducing a new mechanism to the existing market, it is quite likely that only some of the people will start to use it. Even if a mechanism has favorable theoretical properties, assuming that all the agents in the market use it, the existence of outsiders can potentially negatively affect its excellence. Although few papers have carefully considered such problem, CRAB guarantees that each outsider can always enjoy nonnegative payoff gain by joining it even without the full participation of others.

Welfare guarantee To our knowledge, there is no welfare guarantee, such as POA (Roughgarden 2005), applicable to our case: agent-specific affine edge costs with possibly negative constants. In a simple setting with two resources, we evaluate the social welfare attained by CRAB. Notably, the welfare under CRAB is near-optimal when agents' preferences diverge enough. Moreover, the experimental results are also of interest: Our specific initialization rule and choice rule contribute to high welfare under various parameters.

\section{Related work}

The notion of a mediator in a game-theoretic setting has a long history (Aumann 1974; Forges 1986; Myerson 1986;
Bergemann and Morris 2019). Recent works consider several versions of mediation tailored to specific settings, including recommendation system, auction, and routing (Ashlagi, Monderer, and Tennenholtz 2009; Monderer and Tennenholtz 2004; 2009; Kakade et al. 2003; Rozenfeld and Tennenholtz 2007; Rogers and Roth 2014). In particular, as a way to mediate in congestion games, a weak mediator is introduced (Cummings et al. 2015; Kearns et al. 2015). Weak mediators are supposed to be easier to introduce to the real market than one with stronger power (Rozenfeld and Tennenholtz 2007). Thus, this paper focuses on the weak mediator.

Congestion games have also been studied extensively in the field of algorithmic game theory (Nisan et al. 2007). Many versions have been proposed, and our formulation lies in congestion games with player-specific costs (Milchtaich 1996). The existence of pure Nash equilibrium is guaranteed in this game class even when each agent has his own weight (Mavronicolas et al. 2007; Milchtaich 2009; Ackermann, Roglin, and Vocking 2009).

In reality, it is quite likely that only some of the agents will participate in the mechanism after its introduction to the market; it is worth investigating whether outsiders want to start using the mechanism without the full participation of others. However, few papers have carefully considered this safe participation problem (Roth and Shorrer 2018). One notable exception is a routing mediator (Rozenfeld and Tennenholtz 2007), in which the authors let the mediator be informed of the behavior of all agents, including outsiders. Though the assumption is acceptable in their setting, it may not be the case in our large market setting.

\section{Model}

In this section, we introduce our mathematical model of games and equilibrium concepts. We consider a congestion game with incomplete information where agents have private preferences over resources. We call this original oneshot game the underlying game: the strategic situation where agents somehow make their decisions without a mediator. Formally, our underlying game is defined as a tuple $\mathcal{G}=$ $\left\langle\mathcal{I},\left\{\mathcal{A}_{i}, \mathcal{T}_{i}, u_{i}\right\}_{i \in \mathcal{I}}\right\rangle$, where each element is defined as follows:

- Let $\mathcal{I}$ be the finite set of all agents in the market. $|\mathcal{I}|=n$.

- Let $\mathcal{A}_{i} \equiv \mathcal{A}$ be the finite set of resources. The parameter $t_{k}>0$ represents the throughput rate of resource $k{ }^{1}$

- $x_{i} \in \mathcal{A}$ denotes the action of agent $i: x_{i}=k$ iff agent $i$ chooses $k$. We call $x=\left(x_{i}\right)_{i \in \mathcal{I}}$ action profile.

- Let $n_{k}(x)$ be the number of agents choosing $k$ given action profile $x$ :

$$
n_{k}(x):=\left|\left\{i \in \mathcal{I}: x_{i}=k\right\}\right|
$$

\footnotetext{
${ }^{1}$ When resources are trains, for example, we may regard $t_{k}$ as the capacity of train $k$. In such cases, $\lambda_{i}$ represents agent $i$ 's degree of congestion aversion.
} 
- The payoff of each agent $i$ given action profile $x$ is:

$$
u_{i}(x):=\underbrace{v_{i}\left(x_{i}\right)}_{\text {preference term }}-\underbrace{\lambda_{i}}_{\text {opportunity cost }} \cdot \underbrace{\frac{n_{x_{i}}(x)}{t_{x_{i}}}}_{\text {waiting time }}
$$

where $\lambda_{i}>0$ represents the opportunity cost of agent $i$ : the larger $\lambda_{i}$ is, the more congestion-averse agent $i$ is. $v_{i}(k) \in \mathbb{R}$ is agent $i$ 's utility of using resource $k$. The payoff is measured in terms of money.

- $\tau_{i}:=\left(v_{i}(\cdot), \lambda_{i}\right)$ is agent $i$ 's type, which is $i$ 's private information. Let $\mathcal{T}_{i} \equiv \mathcal{T}=\mathbb{R}^{\mathcal{A}} \times \mathbb{R}_{>0}$ be the type space.

\section{Mediator and mediated game}

First, we introduce a mediator to the underlying game described above. The mediator collects private information from each agent and suggests an action to each agent, hoping for mitigating congestion and attaining high social welfare. The introduction of the mediator induces an extensive form game named mediated game; the timing is as follows:

1. Each agent decides whether to use the mediator or not.

2. If he uses the mediator, he reports his type to it.

3. The mediator sends back a recommendation to each agent. Only those who report their type receive the recommendation.

4. All agents in the market, including those who opt-out, simultaneously decide which resource to choose; the payoff is realized.

Each agent can decide whether to utilize the mediator; even when he opts in, he can misreport his type; he is also free to ignore the suggestion. What the mediator can do is just listen to agents' reports and send back a non-binding recommendation. In this sense, we say the mediator is weak.

Formally, mediator is a tuple $\mathcal{M}=\left\langle\left\{R_{i}\right\}_{i \in I}, \mu\right\rangle$, where:

- $R_{i} \equiv R=\{\mathcal{T} \cup\{\perp\}\}$ is the message space of agent $i$ where $\perp$ means opting out of the mediator. Agent $i$ reports $r_{i} \in R_{i}$ to the mediator.

- $\mu: R^{n} \rightarrow \Delta\left(\mathcal{A}^{n}\right)$ is the recommendation rule: it defines how the mediator sends back a recommendation given the reported profile $r=\left(r_{i}\right)_{i} \in R^{n}$.

The mediated game is defined as a tuple $\mathcal{G}^{M}=$ $\left\langle\mathcal{I},\left\{\mathcal{A}_{i}^{M}, \mathcal{T}_{i}, u_{i}\right\}, \mathcal{M}\right\rangle$, where $\mathcal{A}_{i}^{M} \equiv \mathcal{A}^{M}=\mathcal{A}^{\prime} \cup \mathcal{A}^{\perp}$ is the new action space where:

- $\mathcal{A}^{\prime}:=\mathcal{T} \times \mathcal{A}^{\mathcal{A}}$ is the action space of those who opt-in the mediator. The function $f \in \mathcal{A}^{\mathcal{A}}$ determines which action to choose given the suggestion.

- $\mathcal{A}^{\perp}:=\{\perp\} \times \mathcal{A}$ is the action set for those who opt-out. They can freely choose the resource based on their private information.

We do not assume any prior distribution over type space because our solution concept is ex-post Nash equilibrium. This concept of equilibrium is more robust than Bayesian Nash equilibrium, which is usually adopted as a solution concept of an incomplete information game. Weak assumptions on information enables the mediator to be introduced to a variety of situations in the real world.
Definition 1 (Approximate Ex-Post Nash Equilibrium). Given $\epsilon>0$, a pure strategy profile $\sigma:=\left(\sigma_{i}\right)_{i \in \mathcal{I}}$, where $\sigma_{i}: \mathcal{T} \rightarrow \mathcal{A}^{M}$ for each $i$, is $\epsilon$-approximate ex-post pure strategy Nash equilibrium in the mediated game if:

$$
\begin{aligned}
& \forall \tau \in \mathcal{T}^{n} \forall i \in \mathcal{I} \forall x_{i} \in \mathcal{A} ; \\
& u_{i}\left(\sigma_{i}\left(\tau_{i}\right), \sigma_{-i}\left(\tau_{-i}\right)\right) \geq u_{i}\left(x_{i}, \sigma_{-i}\left(\tau_{-i}\right)\right)-\epsilon .
\end{aligned}
$$

Next, we define a specific strategy in the mediated game named good behavior: when following good behavior, an agent uses the mediator, reports his type truthfully, and follows the recommendation. The formal definition is as follows:

Definition 2 (Good behavior). Let id : $\mathcal{A} \rightarrow \mathcal{A}$ be the identity function. The strategy $\sigma_{i}$ of agent $i$ in the mediated game is said to be good behavior if $\sigma_{i}\left(\tau_{i}\right)=\left(\tau_{i}, \mathrm{id}\right)$ for any $\tau_{i} \in \mathcal{T}$.

Suppose that the mediator can compute a Nash equilibrium of the complete information game induced by the reported type profile and send it back to agents. Then, if good behavior forms an ex-post Nash equilibrium in the mediated game, agents can agree to play one specific equilibrium. Moreover, particularly in a congestion game, we can expect that the social welfare under such equilibrium is nearoptimal. Thus, our agenda is to design a mediator with this property.

\section{Credible Recommender based on Agent-made Billboard (CRAB)}

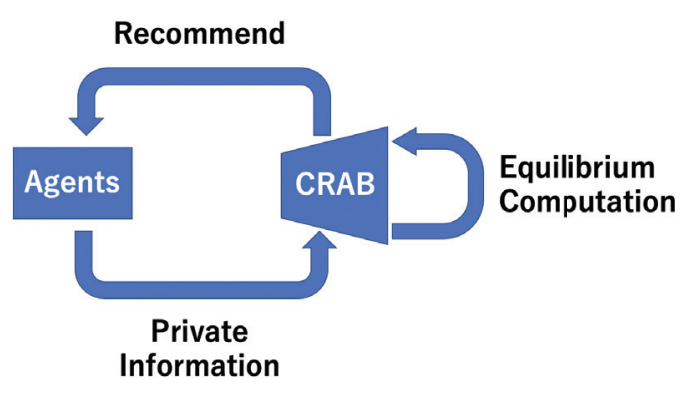

Figure 1: Whole process of CRAB

Now we introduce our mediator, CRAB. Given $R_{i}$, we construct the recommendation rule $\mu$. Suppose that, among $n$ agents in the market, $m$ agents use the mediator. Given the report profile $\left(\tau_{1}, \ldots, \tau_{m}\right)$, the algorithm runs BRD for the induced game played with $m$ agents.

We adopt a version of the famous BRD as our recommendation rule (see Algorithm 1). ${ }^{2}$ In the usual definition of $\mathrm{BRD}$, an initialization rule (which resource agents initially choose) and a choice rule (who moves in each iteration) are undefined, and there is room for exploration. We adopt best as our initialization rule, and max increment as our choice rule: best puts each agent on his best resource initially; max increment chooses the agent who can increase his payoff

\footnotetext{
${ }^{2} \mathrm{BR}_{i}(x) \in \operatorname{argmax}_{x_{i}^{\prime}} u_{i}\left(x_{i}^{\prime}, x_{-i}\right)$ denotes agent $i$ 's best response to $x_{-i}$.
} 
most by a deviation (in case multiple agents meet this criterion, ties are broken at random). This specification simplifies the theoretical analysis of the algorithm. Moreover, it contributes to the better performance of CRAB as we will see in the experimental results section.

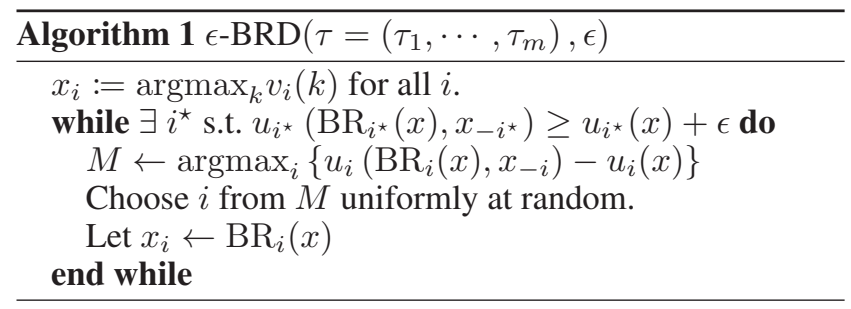

The next proposition guarantees that CRAB always computes equilibrium, and its calculation is fast.

Proposition 1. For any reported type profile and $\epsilon>0$, $C R A B$ computes a pure $\epsilon$-Nash equilibrium in polynomial time (polynomial in the number of agents and resources).

Proof. Let

$$
\Phi(x):=\sum_{i}\left[2 \cdot \frac{v_{i}\left(x_{i}\right)}{\lambda_{i}}+\frac{1}{t_{x_{i}}}\left(n_{x_{i}}(x)+1\right)\right] .
$$

Then, for any $i, x, x_{i}$ and $x_{i}^{\prime}$, we have

$\Phi\left(x_{i}, x_{-i}\right)-\Phi\left(x_{i}^{\prime}, x_{-i}\right)=\frac{2}{\lambda_{i}}\left[u_{i}\left(x_{i}, x_{-i}\right)-u_{i}\left(x_{i}^{\prime}, x_{-i}\right)\right]$.

(This follows from Theorem 6 of Mavronicolas et al. (2007)). We observe that the range of $\Phi$ is polynomial in the number of agents and goods. Moreover, in each iteration, the value of $\Phi$ decreases by at least $2 \epsilon /\left(\min _{i} \lambda_{i}\right)$. The claim follows.

\section{Theoretical Results}

In this section, we focus on the simple two-resource case and prove the theoretical results. Though we only give the proof for the relatively simple cases, in the following section, we confirm that similar results hold in more general settings through numerical experiments. Due to space constraints, we defer all proofs to Appendix.

For our analysis, we pose some assumptions. The first assumption is that the throughput rates go to infinity as the number of agents gets large. Naturally, the capacity of each resource is high in a market with a large population (or we may think that there appear to be many similar resources). This assumption is commonly adopted in the literature of congestion games with a large population. (Feldman et al. 2016).

Assumption 1. For any resource $k, t_{k} \rightarrow \infty$ as $n \rightarrow \infty$.

Next, we assume the behavior of outsiders: each agent chooses his favorite when he opts-out. This can occur as a result of agents' rational decision making when, for example, agents are optimistic enough to believe that their favorites will not be too crowded. More realistic is that they cannot help doing so due to scarce information about each other's preferences.
Assumption 2. If an agent opts out of the mediator, he chooses his favorite resource. ${ }^{3}$

Homogeneous setting There are two resources 1 and 2 . In our homogeneous setting, they have the same throughput rate $t$ in common. Moreover, agents have the same opportunity $\cos t \lambda$.

There are two types of agents, $\tau^{1}$ and $\tau^{2}$ : an agent with $\tau^{k}$ prefers resource $k$ to another. If an agent uses his favorite, his payoff is $v_{1}$ minus the congestion cost; if he uses the lesspreferred one, it is $v_{2}$ minus the congestion cost. The values $v_{1}, v_{2}$ are common across agents. For example, if an agent with type $\tau^{2}$ uses resource 1 , his payoff is $v_{2}-(\lambda / t) \cdot n_{1}$.

First, we can show that good behavior forms an equilibrium with $C R A B$, assuming the participation of all agents in the market.

Theorem 1 (Implementation [homogeneous case]). Assume that all agents opt-in the mediator. In a homogeneous setting, good behavior forms $(\lambda / t)$-approximate ex-post Nash equilibrium in the mediated game. The approximation factor tends to zero as the market gets large under Assumption 1.

Proof sketch. Let $s_{k}$ be the number of agents with $\tau^{k}$ ( $k \in$ $\{1,2\})$. Wlog, assume $s_{1} \geq s_{2}$. The simplicity of the setting allows us to analyze the behavior of CRAB in detail. We can show that what our BRD does is to move agents with $\tau^{1}$ from 1 to 2 until it reaches equilibrium. By this property, we can divide the proof into subtle cases and obtain the statement. See the Appendix for further details.

Next, we consider the case where only some of the agents currently uses the mediator. We show that it is safe for each outsider to start using CRAB.

Theorem 2 (Safe participation [homogeneous case]). Suppose that all users of CRAB follow good behavior. Then, in a homogeneous setting, each outsider enjoys non-negative payoff gain when joining the mediator under Assumption 2.

Lastly, we conduct a welfare analysis. It is well known that the welfare under a pure Nash equilibrium in congestion games and its variants are often near-optimal (POA is close to 1 (Roughgarden 2016)); it is naturally expected that the same is true in the settings with individual preference, $v_{1}$ and $v_{2}$.

Below, we additionally assume that throughput rates $\left(t_{k}\right)_{k}$ cannot increase as rapidly as population $n$. This seems natural given the law of diminishing marginal returns.

Assumption 3. $t_{k}=o(n)$ for any $k$.

The following theorem provides the welfare guarantee: the price of mediation (POM) is the ratio between the social welfare under the good behavior equilibrium and the first-best, which is the maximized social welfare, ignoring the incentive constraints. Formally:

$$
\mathrm{POM}=\frac{\sum_{i} u_{i}\left(\mu\left(\left(\tau_{i}\right)_{i}\right)\right)}{\max _{x} \sum_{i} u_{i}(x)}
$$

\footnotetext{
${ }^{3}$ We use Assumption 2 only when proving Theorem 2 and 5.
} 
Let $s \in[0,1]$ be the fraction of agents with $\tau^{1}$. Wlog, we assume $s \in[1 / 2,1]$. Note that the social welfare can be both positive and negative in general. However, if $n$ is so large that $v_{1}<\left(2 s^{2}-2 s+1\right) n c$, we can show that the first-best takes a negative value (see the Appendix for details). As we focus on the large market, we restrict our attention to such case where POM is more than or equal to 1 .

We can compute the upper bound of POM in the large market explicitly (see the Appendix for the details):

Theorem 3 (POM bound in the large market). For a sufficiently large market, POM is bounded from above by:

$$
\frac{1}{4}(2 s-1)^{4}+\frac{1}{2}(2 s-1)^{3}+\frac{13}{4}(2 s-1)^{2}+1 .
$$

POM attains its minimum 1 at $s=1 / 2$ and attains its maximum 5 at $s=1$. It is increasing on $[1 / 2,1]$. Moreover, it is convex and increases slowly when $s$ is not close to 1 . This means that as long as the agents' preferences diverge enough, the social welfare attained at the equilibrium induced by the mediator is close to the firstbest. Table 3 expresses the exact POM value at each $s=$ $\{0.5,0.6,0.7,0.8,0.9,1.0\}$.

\begin{tabular}{|c||c|c|c|c|c|c|}
\hline$s$ & 0.5 & 0.6 & 0.7 & 0.8 & 0.9 & 1.0 \\
\hline POM & 1.00 & 1.13 & 1.56 & 2.31 & 3.44 & 5.00 \\
\hline
\end{tabular}

Table 3: POM increases slowly as long as $s$ is not very close to 1 .

Heterogeneous setting Our heterogeneous setting is more general than the homogeneous setting in two senses: First, resources differ in throughput rates, or $t_{1} \neq t_{2}$. Second, agents have different opportunity costs $\lambda_{H}$ and $\lambda_{L}$, where $\lambda_{H}>\lambda_{L}$.

There are four types of agents: $\left(\tau^{1}, \lambda_{H}\right),\left(\tau^{1}, \lambda_{L}\right)$, $\left(\tau^{2}, \lambda_{H}\right)$, and $\left(\tau^{2}, \lambda_{L}\right)$. Again, agents with $\tau^{k}$ prefer resource $k$. For instance, the payoff of an agent with $\left(\tau^{2}, \lambda_{H}\right)$ choosing resource 1 is $v_{2}-\left(\lambda_{H} / t_{1}\right) n_{1}$.

We have the generalizations of Theorem 1 and Theorem 2 for this setting:

Theorem 4 (Implementation [heterogeneous case]). Assume that all agents opt-in the mediator. In a heterogeneous setting, good behavior forms $\left(\lambda_{H} / \min \left\{t_{1}, t_{2}\right\}\right)$ approximate ex-post Nash equilibrium in the mediated game. The approximation factor tends to zero as the market gets large under Assumption 1.

Theorem 5 (Safe participation [heterogeneous case]). Suppose that all users of CRAB follow good behavior. Then, in a heterogeneous setting, each outsider enjoys non-negative payoff gain when joining the mediator under Assumption 2.

In the following section, we confirm that the results of these theorems can be extended to more general settings with experiments. In particular, Theorem 4 leads us to guess about the upper-bound of the payoff gain by deviation: we guess the bound is $\bar{\lambda} / \underline{t}$, where $\bar{\lambda}:=\max _{i} \lambda_{i}$ and $\underline{t}=$ $\min _{k} t_{k}$, which also tends to zero under Assumption 1.

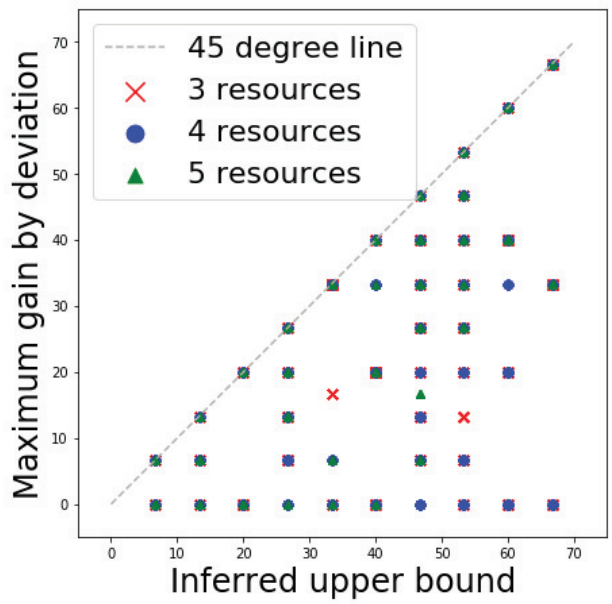

Figure 2: The maximum payoff gain by deviation from good behavior is bounded from above by the value inferred from Theorem 4, even with more than two resources.

\section{Experimental Results}

In this section, we show the results of our series of experiments. The experimental details, including the precise parameter settings, are provided in the Appendix.

\section{Extensions to general cases}

We confirm that the theoretical results we have shown in the previous section can be extended to more general cases.

First of all, we check whether the payoff gain by deviation from good behavior is bounded from above. From Theorem 4 , we guess that the upper bound should be $\bar{\lambda} / \underline{t}$ where $\bar{\lambda}:=\max _{i} \lambda_{i}$ and $\underline{t}=\min _{k} t_{k}$. We conduct simulations in a large number of different parameter settings with 3 or more resources to check this hypothesis.

Figure 2 summarizes the results. The horizontal line corresponds to the inferred upper bound $\bar{\lambda} / \underline{t}$, and the vertical line is the maximum payoff gain. Each instance represented by a dot comes below the 45-degree line, which indicates the inferred upper bound is valid when the number of resources is more than two.

We also check if the safe participation property holds in the more general cases. We conduct simulations in various parameter settings with up to 10 resources.

The first remarkable observation is that no outsider decreases his payoff by entering the mediator regardless of the market size and the participation rate. Furthermore, we find two general trends; more outsiders get strictly positive payoff gains by joining the mediator as:

1. the participation rate gets higher for any market size; and

2. the number of existing agents gets larger for any participation rate.

Figure 3 summarizes the simulation results. These features suggest that $C R A B$ can benefit the individual more when the market size is large and more people use CRAB. 


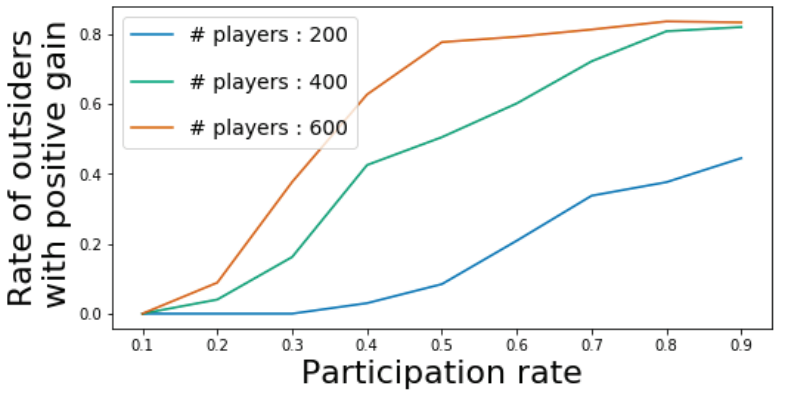

Figure 3: Plot the average rate of outsiders with positive gain at each participation rate. We take an average of 20 experiments with 4 resources.

\section{Close study of the detail of BRD}

With the actual implementation in mind, we analyze how the details of BRD affect the performance of the mediator. Our objective is to make the mediator faster, more robust, and more efficient.

In order to define BRD precisely, we need to fix the choice rule and the initialization rule, neither of which the usual definition of $\mathrm{BRD}$ precisely determines. The choice rule specifies who moves at each step of BRD. Here, we consider two options: (1) to pick the agent with the maximum payoff gain (max increment), or (2) to pick the one with the minimum payoff gain (min increment). As for the initialization rule, we compare three alternatives: initially, all agents choose (1) their best preferred resources (best), (2) their worst preferred ones (worst), and (3) the same one resource (oneside). The BRD used by CRAB adopts the pair of rules max increment and best. We call this pair the $C R A B$ rule. The CRAB rule not only simplifies the theoretical analysis, as shown in Appendix, but also enables CRAB to perform better in practice.

We conduct experiments in a two-resource setting. We try 2, 160 parameters made from reasonable values. For all pairs of rules, we compare the realized social welfare, which is the summation of the payoffs of all agents, and the iteration numbers required to reach equilibrium.

\begin{tabular}{c|ccc}
\hline & BEST & WORST & ONESIDE \\
\hline MAX INCREMENT & 0.904 & 0.826 & 0.830 \\
MIN INCREMENT & 0.823 & 0.812 & 0.830 \\
\hline
\end{tabular}

Table 4: Equilibrium selection. CRAB rule (top, left) gives the highest rate of obtaining the best equilibrium among the six pairs. The different rules may converge to the same best equilibrium; the sum of all cell values may not be 1 .

Each cell in Table 4 indicates the fraction of parameter sets in which a corresponding pair of rules attain the highest social welfare among the six rules. The CRAB rule obtains the highest social welfare in $90 \%$ of the parameters, which outperforms the other pairs of rules.

In the experiments, we observe that the max increment

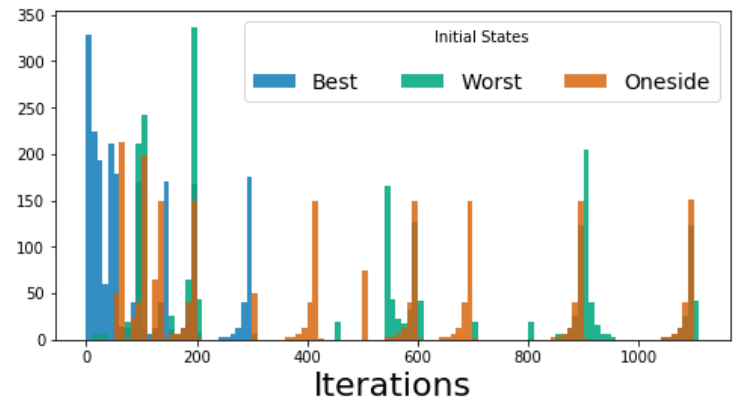

Figure 4: Histogram of necessary iterations for each initial state. We consider only the max increment picking rule.

demands fewer iterations than the min increment. We fix the picking rule to the former and compare the required steps for each initialization rule. Figure 4 gives the histogram of the number of iterations to reach an equilibrium: the CRAB rule requires a fewer number of iterations than the others.

\section{Discussion and Future Directions}

We prove our theoretical results for the two-resource case. One natural extension is to provide the proofs for general cases; for the moment, we observe that the results are likely to hold just experimentally. It is worth exploring whether similar results hold when (1) there are more than two resources, (2) the number of sets of opportunity costs is more than two, or (3) the values of the utility of resources differ among agents (NB: we assume that agents have $v_{1}$ and $v_{2}$ in common).

We are now planning to implement CRAB as a web application in a real crowded market to evaluate its practical performance (see also the Appendix). Such empirical work is also one possible research direction.

However, to this end, we need to resolve the report cost problem, the classical problem in the mechanism design literature: it is too demanding for agents to report their preferences over all the alternatives. It is unrealistic to require people to rank all available alternatives and report that ordering; it is not only burdensome but also cognitively challenging.

Another problem regarding report costs, which is unique to the congestion problem, is that the mediator needs to know not only the agents' valuations about resources, $v_{i}(\cdot)$, but also their opportunity costs $\lambda_{i}$. We cannot expect them to report their opportunity costs in addition to the preference list. It is nearly impossible to ask questions like: "Which do you prefer, a) resource 1 with a waiting time of 10 minutes, b) resource 2 with a waiting time of 30 minutes, or c) resource 3 with a waiting time of 25 minutes?"

We believe that the mediator incorporating statistical analysis enables us to handle the report cost problems. CRAB, in real markets, estimates the true parameter values and retrieves the full preference lists, utilizing the data collected through the repeated use of the application. This topic must be of interest from both theoretical and practical perspectives. 


\section{References}

Ackermann, H.; Roglin, H.; and Vocking, B. 2009. Pure Nash Equilibria in Player-Specific and Weighted Congestion Games. Theoretical Computer Science 410(17):1552 $-1563$.

Allcott, H., and Rogers, T. 2014. The Short-Run and LongRun Effects of Behavioral Interventions: Experimental Evidence from Energy Conservation. American Economic Review 104(10):3003-37.

Anderson, M. L.; Lu, F.; Zhang, Y.; Yang, J.; and Qin, P. 2016. Superstitions, Street Traffic, and Subjective Wellbeing. Journal of Public Economics 142:1-10.

Ashlagi, I.; Monderer, D.; and Tennenholtz, M. 2009. Mediators in Position Auctions. Games and Economic Behavior 67(1):2-21.

Aumann, R. J. 1974. Subjectivity and Correlation in Randomized Strategies. Journal of Mathematical Economics 1(1):67-96.

Bergemann, D., and Morris, S. 2019. Information Design: A Unified Perspective. Journal of Economic Literature 57(1):44-95.

Cummings, R.; Kearns, M.; Roth, A.; and Wu, Z. S. 2015. Privacy and Truthful Equilibrium Selection for Aggregative Games. In Proceedings of the 11th International Conference on Web and Internet Economics - Volume 9470, 286-299.

Feldman, M.; Immorlica, N.; Lucier, B.; Roughgarden, T.; and Syrgkanis, V. 2016. The Price of Anarchy in Large Games. In Proceedings of the Forty-eighth Annual ACM Symposium on Theory of Computing, 963-976.

Forges, F. 1986. An Approach to Communication Equilibria. Econometrica 54(6):1375-1385.

Gibson, M., and Carnovale, M. 2015. The Effects of Road Pricing on Driver Behavior and Air Pollution. Journal of Urban Economics 89:62-73.

Ieong, S.; McGrew, R.; Nudelman, E.; Shoham, Y.; and Sun, Q. 2005. Fast and Compact: A Simple Class of Congestion Games. In Proceedings of the 20th National Conference on Artificial Intelligence - Volume 2, 489-494.

Ito, K.; Ida, T.; and Tanaka, M. 2018. Moral Suasion and Economic Incentives: Field Experimental Evidence from Energy Demand. American Economic Journal: Economic Policy 10(1):240-67.

Kakade, S.; Kearns, M.; Langford, J.; and Ortiz, L. 2003. Correlated Equilibria in Graphical Games. In Proceedings of the 4th ACM Conference on Electronic Commerce, 42-47.

Kearns, M.; Pai, M.; Roth, A.; and Ullman, J. 2014. Mechanism Design in Large Games: Incentives and Privacy. In Proceedings of the 5th Conference on Innovations in Theoretical Computer Science, 403-410.

Kearns, M.; Pai, M. M.; Rogers, R.; Roth, A.; and Ullman, J. 2015. Robust Mediators in Large Games. arXiv:1512.02698 [cs]. arXiv: 1512.02698.

Kreindler, G. E. 2018. The Welfare Effect of Road Congestion Pricing: Experimental Evidence and Equilibrium Implications. MIT Working paper.
Mavronicolas, M.; Milchtaich, I.; Monien, B.; and Tiemann, K. 2007. Congestion Games with Player-Specific Constants. In Kučera, L., and Kučera, A., eds., Mathematical Foundations of Computer Science 2007, Lecture Notes in Computer Science, 633-644.

Milchtaich, I. 1996. Congestion Games with PlayerSpecific Payoff Functions. Games and Economic Behavior 13(1):111-124.

Milchtaich, I. 2009. Weighted Congestion Games with Separable Preferences. Games and Economic Behavior 67(2):750-757.

Monderer, D., and Tennenholtz, M. 2004. KImplementation. Journal of Artificial Intelligence Research 21:37-62.

Monderer, D., and Tennenholtz, M. 2009. Strong Mediated Equilibrium. Artificial Intelligence 173(1):180-195.

Myerson, R. B. 1986. Multistage Games with Communication. Econometrica 54(2):323-358.

NAVITIME, J. 2018. Hatsushisan! Man-in Densha No Keizai Sonshitsu Wa Nenkan 3240 Okuen. (October 18, 2018) Available at TOYO KEIZAI ONLINE: https://toyokeizai.net/articles/-/243263.

Nisan, N.; Roughgarden, T.; Tardos, E.; and Vazirani, V. V., eds. 2007. Algorithmic Game Theory. Cambridge University Press.

Okano, H.; Ota, K.; and Hirota, M. 2017. Estimation of the Railway Congestion. 55th Committee of Infrastructure Planning and Management.

Palaiopanos, G.; Panageas, I.; and Piliouras, G. 2017. Multiplicative Weights Update with Constant Step-Size in Congestion Games: Convergence, Limit Cycles and Chaos. In Advances in Neural Information Processing Systems 30. 5872-5882.

Rogers, R. M., and Roth, A. 2014. Asymptotically Truthful Equilibrium Selection in Large Congestion Games. In Proceedings of the Fifteenth ACM Conference on Economics and Computation, EC' $14,771-782$.

Roth, B., and Shorrer, R. I. 2018. Making it Safe to Use Centralized Marketplaces: Dominant Individual Rationality and Applications to Market Design (October 1, 2018). Available at SSRN: https://ssrn.com/abstract=3073027.

Roughgarden, T. 2005. Selfish Routing and the Price of Anarchy. The MIT Press.

Roughgarden, T. 2016. Twenty Lectures on Algorithmic Game Theory. Cambridge University Press.

Rozenfeld, O., and Tennenholtz, M. 2007. Routing Mediators. In Proceedings of the 20th International Joint Conference on Artifical Intelligence, IJCAI'07, 1488-1493.

Vickrey, W. S. 1963. Pricing in Urban and Suburban Transport. American Economic Review Proceedings 452-65. 